\title{
Scattering and Absorption Performance of a Microsize Graphene-Based Parabolic Reflector in the THz Range Illuminated by a Complex Line Source
}

\author{
Taner Oğuzer \\ Dept. Electrical and Electronics Engineering \\ Dokuz Eylul University, Buca, 35160 Izmir, Turkey \\ taner.oguzer@deu.edu.tr
}

\author{
Ayhan Altintas \\ Dept. Electrical and Electronics Eng., Bilkent University \\ 06800 Ankara, Turkey \\ altintas@ee.bilkent.edu.tr
}

\begin{abstract}
The scattering and absorption characteristics of a twodimensional (2-D) parabolic reflector made of graphene and placed in the free space is simulated using the Method of Analytical Regularization (MAR) technique. Reflector is illuminated by a complex magnetic line source having a directive beam-like antenna pattern and placed in the geometrical focus of reflector. The total absorbed power and forward and backward directivities are computed. The surface plasmon (SP) resonances are observed. Besides, the scattering performance of the reflector is studied in dependence of the chemical potential of the graphene.
\end{abstract}

Keywords-electromagnetic scattering; graphene reflector.

\section{INTRODUCTION}

The past 10 years have witnessed sharp growth of interest in graphene as a non-conventional material, which is a very thin (sometimes called single-atom) layer with adjustable electrical and chemical properties [1-3]. Its conductive properties are characterized with the aid of surface impedance $Z$ that has inductive nature (its imaginary part is negative if the time dependence is chosen as $\exp (-i \omega t))$. Thanks to that fact, a sheet of graphene can support the surface plasmon (SP) guided wave [4]. If graphene is patterned, i.e. shaped as finite sample, then the SP wave can bounce back and forth between the edges of graphene sample. This leads to the formation of standing waves, i.e. to Fabry-Perot type resonances called SPmode resonances. What is remarkable, SP resonances are observed both in the infrared range - if graphene sample has nanoscale dimensions and in the terahertz range - if it has microscale dimensions. Today this effect is used in the design of mid-infrared bio-chemical sensor systems but it can be also used in some quasi-optical elements in the THz range.

Another interesting property of graphene relates to the observation that its electron conductivity can be changed in wide range by using electrostatic biasing field which changes graphene's chemical potential. To realize this electric tuning in practical circumstances, researchers usually place a sheet of graphene on or into a thin dielectric substrate. Still freestanding (called suspended) graphene sheets are also investigated due to their smaller dependence on the chemical degradation. Thus, any electromagnetic-wave system or device that incorporates a graphene element is potentially an electrically tunable system or device.

The most wide spread today microscopic model of graphene conductivity is based on the Kubo formalism [1-3]. From the viewpoint of the modeling, important observation is that in the finite graphene samples larger than $100 \mathrm{~nm}$ in size, the edge effects can be neglected and the electron conductivity (and hence the surface impedance) can be characterized using the model developed for infinite graphene.

One of the less studied topics around potential applications of graphene is the characterization of micro-size reflectors made of graphene. In recent papers [5, 6], we have analyzed a 2-D model of finite microsize graphene reflector located in the free space and illuminated by a plane wave incident along the plane of symmetry. Both the far-field scattering characteristics such as the total scattering cross-section and the near-field effects such as the focusing were investigated as a function of frequency and reflector's geometry. This has enabled us to conclude that efficient performance of graphene reflector can be expected only in the lower part of $\mathrm{THz}$ range, because of rapid growth of $|Z|$ with frequency.

The aim of this paper is to extend the studies of 2-D parabolic graphene reflector in the free space to the case of illumination by a directive feed, i.e. the same antennas as in $[5,6]$ however in the transmission regime. We will restrict our analysis to the H-polarization case.

As known, if the surface impedance of graphene is given, then the analysis of cylindrical reflector can be considered as an electromagnetic boundary value problem (BVP). It involves the Helmholtz equation, the resistive-sheet boundary condition [7,8], the edge condition, and the radiation condition at infinity. Taken together, these conditions provide the uniqueness of the solution of BVP [9].

Full-wave modeling of reflector antennas can be done using many numerical methods. For engineering aims, the finite- 
difference time-domain (FDTD) method is frequently chosen. However it is not a good choice for large, quasi-optical size, reflectors as it suffers of huge domain to be discretized and hence leads to unrealistic computation time. The method of moments (MoM) technique can also be applied to arbitrary geometries. However, by using the local basis and testing functions, its accessible accuracy is quite low (2-3 digits) even for a few-lambda reflectors. The high frequency techniques like GO, PO, PTD are fast however neglect fine details of wave fields.

As we are interested in fundamental wave effects, we analyze the graphene reflector performance using MAR (see, for instance, $[10,11])$. To invert analytically the most singular part of the associated singular-integral-equation (SIE) operator, we follow our earlier works on the H-wave scattering by PEC and resistive 2-D reflectors $[12,13]$. This involves a specialized discretization technique known as the Riemann-Hilbert Problem (RHP) method [8]. The result is a Fredholm second-kind infinite matrix equation that provides a convergent numerical solution. The SIE-MAR technique enables very fast and accurate full-wave analysis of the reflector problems with various incident field functions.

As graphene's impedance depends on frequency, it can be expected that graphene reflectors performance should also depend on frequency more profoundly than for their PEC analogs. The role of the SP resonances is also a matter of study. Here, we can keep in mind the scattering and absorption of $\mathrm{THz}$ waves by flat graphene strips and finite graphene-strip gratings has already shown the presence of SP resonances, especially strong in the lower part of THz range [14-19].

In the present study, the feed is taken as a complex-sourcepoint (CSP) magnetic line-current field. CSP is a model of aperture feed such as a small horn antenna. Therefore the output characteristics are the angular far-field pattern and the partial (i.e. forward and backward) directivities of CSP excited graphene reflector system. As mentioned, graphene impedance is modeled using the Kubo formulas and the boundary condition at reflector's surface is the resistive-sheet condition $[7,8]$ with frequency-dependent complex resistivity.

\section{FORMULATION}

The cross-sectional geometry of a 2-D parabolic graphene reflector illuminated in the frontal manner by a CSP magnetic line source is presented in Fig. 1. Here, the reflector contour $M$ is completed with a circular arc $S$ to a closed contour denoted as $C$ in such way that the curvatures of two arcs are the same at the junctions, i.e. at reflector's edge points. Such a smooth contour $C$ is necessary for the discretization leading to the regularized matrix equation of the Fredholm second-kind nature.

Assume that the CSP feed's center of the aperture is located at arbitrary point having the real position vector $\vec{r}_{0}\left(x_{0}, y_{0}\right)$ and the complex shift is achieved by adding a complex vector $i \vec{b}$.

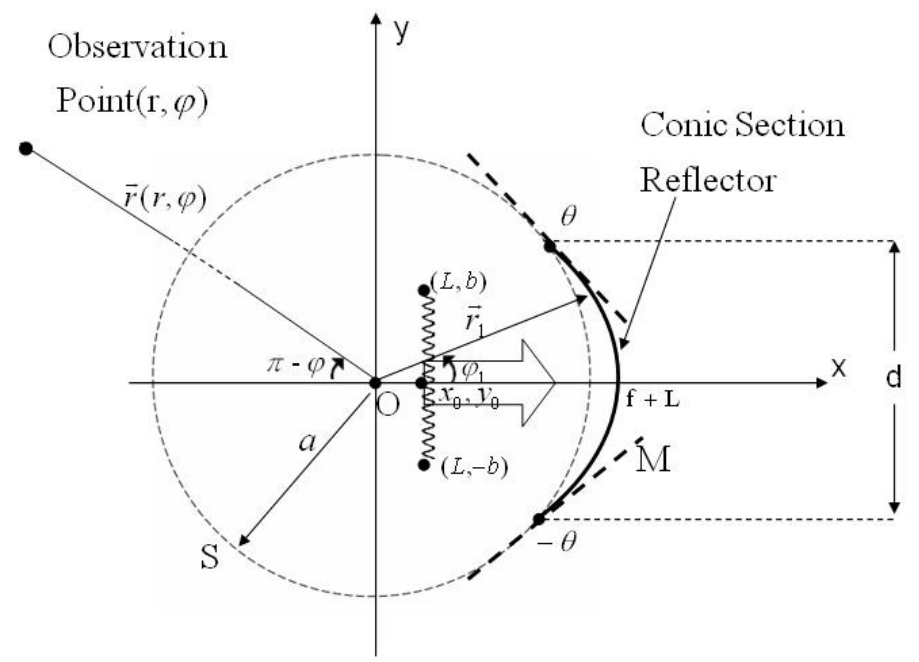

Figure 1. Cross-sectional geometry of graphene parabolic reflector fed by a CSP feed placed at the line of symmetry.

Then the resultant complex position vector becomes $\vec{r}_{s}=\vec{r}_{0}+i \vec{b}$. The line source located at this complex position radiates a directive antenna pattern according to the following equation:

$$
H_{z}^{i n}=H_{0}^{(1)}\left(k\left|\bar{r}-\bar{r}_{s}\right|\right),
$$

where $k$ is the free-space wavenumber. Note that function (1) has two branch points which should be connected with a branch cut (see Fig. 1). The direction of radiation of CSP feed is governed by the vector $\vec{b}$ and can be arbitrary. Still, in computations, we will assume that CSP is looking at the center of reflector as such orientation provides obviously better performance than other, non-symmetric illuminations. Guided by the similar considerations, we will also assume that the middle point of CSP feed is located at the geometrical focus of the parabolic reflector.

The resistive boundary condition first appeared as a model of either a thinner than skin depth sheet of metal or a very thin dielectric layer [7, 8]. It combines together two equations for the field tangential components,

$$
\left[\vec{E}_{\text {tan }}^{+}+\vec{E}_{\text {tan }}^{-}\right] / 2=Z \underbrace{\vec{n} \times\left[\vec{H}_{z}^{+}-\vec{H}_{z}^{-}\right]}_{\vec{J}_{\mathrm{t}}}, \quad \vec{E}_{\tan }^{+}=\vec{E}_{\tan }^{-} \quad \vec{r} \in M,
$$

where the superscripts "- " and "+" relate to the concave and the convex sides of reflector, respectively, and the unit normal vector $\vec{n}$ is directed to the concave side of the reflector. Note that the jump in magnetic field is the electric surface current density (unknown function).

For a graphene sheet, the resistivity or, equivalently, the surface impedance, is connected to the graphene conductivity $\sigma$ as $Z=1 / \sigma$. The Kubo formulas for the conductivity can be found in many textbooks (see [3]). Although this result was derived for the infinite planar graphene layer, it can also be approximately used for the finite smoothly curved surfaces.

The boundary condition (2) leads to the following the electric field integral equation (EFIE) for the surface current: 


$$
\begin{aligned}
Z J_{\mathrm{t}}= & -\frac{i Z_{0}}{k} \frac{\partial H_{z}^{i n}}{\partial n}+\frac{i Z_{0}}{k} \frac{\partial}{\partial l} \int_{M}\left[\frac{\partial}{\partial l^{\prime}} J_{t}\left(\vec{r}^{\prime}\right)\right] G\left(\vec{r}, \vec{r}^{\prime}\right) d l^{\prime} \\
& +i k Z_{0} \int_{M} J_{t}\left(\vec{r}^{\prime}\right) \cos \left[\xi(\vec{r})-\xi\left(\vec{r}^{\prime}\right)\right] G\left(\vec{r}, \vec{r}^{\prime}\right) d l^{\prime},
\end{aligned}
$$

where the scalar Green's function $G$ is a Hankel function of the zero order and first kind satisfying the radiation condition; i.e. $G\left(\vec{r}, \vec{r}^{\prime}\right)=(i / 4) H_{0}^{(1)}\left(k_{o} R\right), \quad R=\left|\vec{r}-\vec{r}^{\prime}\right|$. Assume that the curve $M$ can be parameterized using the equations $x=x(\varphi), y=y(\varphi)$ in terms of polar angle, $\varphi, 0 \leq|\varphi| \leq \theta$.

To proceed with the MAR-based formulation, all functions should be expended in terms of the Fourier series. Furthermore, we add and subtract the similar functions from the originally given ones. These are the functions at the full circle of the closed contour $C$ and introduced as follows.

$$
\begin{aligned}
H\left(\varphi, \varphi^{\prime}\right) & =H_{0}^{(1)}(k R)-H_{0}^{(1)}\left(2 k a \sin \left(\left|\varphi-\varphi^{\prime}\right| / 2\right)\right), \\
S\left(\varphi, \varphi^{\prime}\right)= & \cos \left(\xi(\varphi)-\xi\left(\varphi^{\prime}\right)\right) \beta(\varphi) \beta\left(\varphi^{\prime}\right) H_{0}^{(1)}\left(k\left|\vec{r}(\varphi)-\vec{r}^{\prime}\left(\varphi^{\prime}\right)\right|\right) \\
& -\beta^{2}(\varphi) H_{0}^{(1)}\left(2 k a \sin \left(\left|\varphi-\varphi^{\prime}\right| / 2\right)\right) .
\end{aligned}
$$

The functions $H$ and $S$ have also continuous first derivatives, and their second derivatives with respect to $\varphi$ and $\varphi$ ' have only logarithmic singularity and hence belong to $L_{2}$. Therefore their Fourier coefficients tend to zero with larger indices and can be efficiently computed by the Fast Fourier Transform algorithm.

In (4), we use the differential lengths in the tangential direction at any point on $M$ given by $\partial l=a \beta(\varphi) \partial \varphi$ and $\partial l^{\prime}=a \beta\left(\varphi^{\prime}\right) \partial \varphi^{\prime}$, respectively, while $\beta(\varphi)=r(\varphi) /[a \cos \gamma(\varphi)]$. Besides, $\xi(\varphi)$ is the angle between the normal on $M$ and the $\mathrm{x}$-direction, and $\gamma(\varphi)$ is the angle between the normal and the radial direction. By extending the surface-current density $J_{t}$ with zero value to $S$, we extend SIE (3) to complete contour $C$ made of $S$ and $M$ and thus obtain a set of dual equation.

Eventually we discretize these dual equations on reflector $M$ and on aperture $S$ and cast them to a dual series equation. They can be treated with MAR approach using the RHP technique and yield an algebraic equation system $[8,12,13]$. This infinite matrix equation is of the Fredholm second kind which guarantees the existence of the exact solution in $L_{2}$ and also the convergence of approximate solutions, found with finite truncation numbers, to that exact solution.

\section{SCATTERING AND ABSORPTION CHARACTERISTICS}

As we would like to study the far-field characteristics of a graphene reflector illuminated by the in-GO-focus H-polarized CSP feed, we express the far zone total radiated field as follows:

$$
H_{z}(\vec{r})=\left[\Phi_{i n}(\varphi)+\Phi_{s c}(\varphi)\right](2 / i \pi k r)^{1 / 2} e^{i k r},
$$

where

$$
\Phi_{i n}(\varphi)=\exp [k b \cos (\phi-\beta)],
$$

and $\Phi_{s c}(\varphi)$ is found from the solution of IE (3) (see [12, 13])

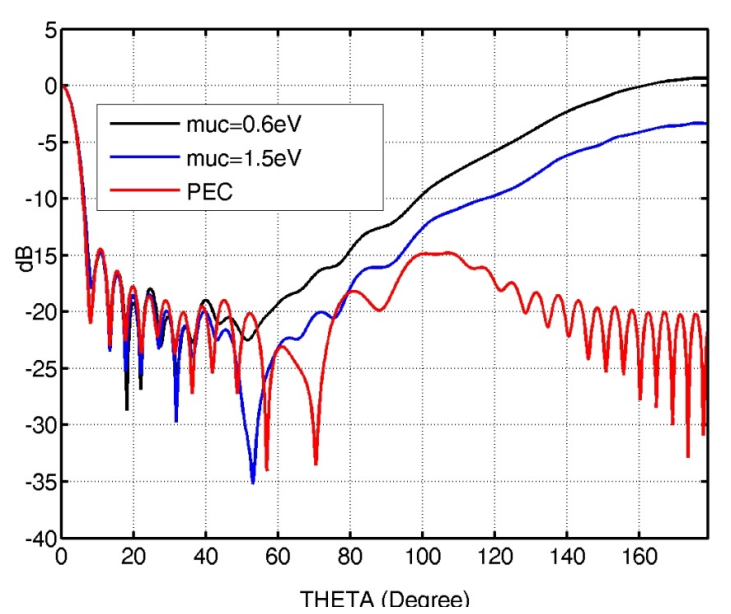

Figure 2. Normalized radiation patterns for a parabolic reflector front-fed by in-GO-focus CSP feed. The problem parameters are given as $f / d=0.4, d=$ $500 \mu \mathrm{m}$ and frequency is $8 \mathrm{THz}, k b=3, T=300 \mathrm{~K}, \tau=1 \mathrm{ps}$.

as the angular part of the far-field asymptotic of the scattered field function,

$$
H_{z}^{s c}(\bar{x}, \bar{y})=\int_{-\theta}^{\theta} \tilde{J}_{t}\left(\varphi^{\prime}\right) \frac{\partial G}{\partial n^{\prime}} a \beta\left(\varphi^{\prime}\right) d \varphi^{\prime},
$$

where the $\bar{x}$ and $\bar{y}$ parameters are the observation point coordinates. In computations, we will use $\beta=0$ to provide symmetric illumination of reflector.

The total power radiated by the CSP feed in the presence of reflector is given by

$$
P_{\text {rad }}=\frac{Z_{0}}{\pi k} \int_{0}^{2 \pi}\left|\Phi_{i n}(\varphi)+\Phi_{s c}(\varphi)\right|^{2} d \varphi,
$$

where $Z_{0}$ is the free-space impedance. Note that $P_{r a d}$ is not the same quantity as the power $\left(P_{t o t}\right)$ taken from the feed because of absorption in imperfect graphene reflector. The total taken power can be found from the Poynting theorem [20] adapted to the CSP feed and applied to the studied antenna (see $[13,21]$ as examples of such derivation).

Then the power absorbed by graphene reflector surface can be found as follows:

$$
P_{a b s o r b e d}=P_{t o t}-P_{r a d} \text {. }
$$

Note that in the absence of reflector (i.e. in the free space), the considered here CSP feed radiates the averaged per period power $P_{0}=\left(2 Z_{0} / k\right) I_{0}(2 k b)$, where $I_{0}(\cdot)$ is the modified Bessel function. In computations, it is reasonable to normalize all power quantities by this value.

\section{NUMERICAL RESULTS}

The numerical accuracy and convergence of the given formulation had already been verified in $[12,13]$ in the presence of the CSP illumination. In the present study, we would like to analyze a reflector made of graphene and make an estimation of the effects of the partial transparency and the SP resonances. In Fig. 2, the normalized radiation pattern is plotted for various chemical potential $\left(\mu_{c}\right)$ values. 

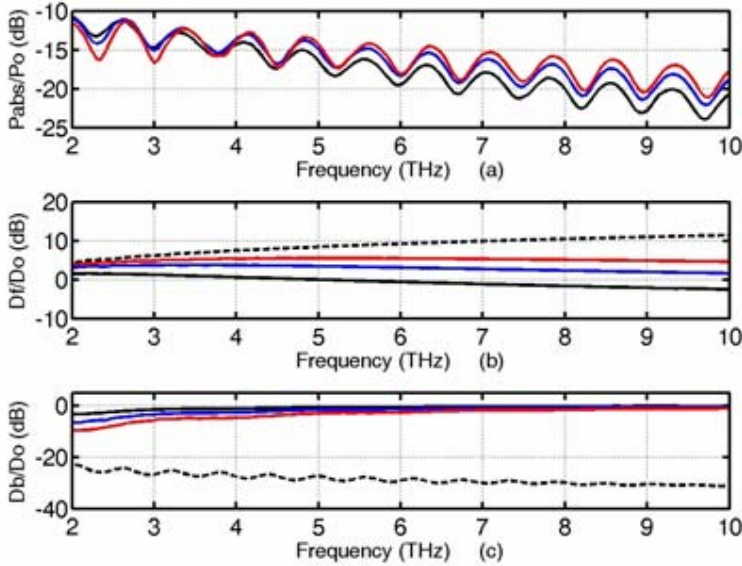

Figure 3. From top to bottom: normalized absorbed power, forward directivity and backward directivity of a CSP-fed graphene reflector as a function of the frequency in the $\mathrm{THz}$ range. Black curves are for $\mu_{c}=0.6 \mathrm{eV}$, blue curves are for $\mu_{c}=1 \mathrm{eV}$, and red curves are for $\mu_{c}=1.5 \mathrm{eV}$, all with $T=300 \mathrm{~K}$ and $\tau=1 \mathrm{ps}$. The dashed curves correspond to the PEC reflector. The reflector parameters are as follows: focal parameter $f / d=0.4$, size $d=500 \mu \mathrm{m}$, and the feed parameter is $k b=3$.

It is seen that higher values of chemical potential lead to the lower values of the surface impedance of graphene that makes it better conducting and hence the curves get closer to the PEC reflector case. The reduction of $\mu_{c}$ gradually increases the transparency of reflector (remember that graphene is transparent in the visible range). Therefore the spillover lobe is visible only if $\mu_{c}$ is large and if it is not, the backward space of reflector shows the radiation pattern close to $\Phi_{\text {in }}(\varphi)$ of the CSP in free space, given by (3).

In Fig. 3, we present the absorbed power and the forward and backward directivities as a function of the frequency. The oscillations in the absorbed power are due to the interference of the waves scattered by the edges of reflector. Besides, if one increases the chemical potential, $\mu_{c}$, then certain amount of increase is seen in the absorbed power. The forward directivity is getting higher with the higher chemical potential values. As mentioned above, if the frequency is getting higher then a graphene reflector becomes more and more transparent because its surface impedance $Z$ increases almost linearly, with the dominant imaginary part, and finally it exceeds the free-space impedance value (see [3,14-16]). Therefore, the linear increase in the forward directivity is restricted to lower $\mathrm{THz}$ range and is replaced with reduction if the frequency gets above $2 \mathrm{THz}$ for realistic values of the chemical potential.

\section{CONCLUSIONS}

A 2-D graphene reflector in the free space, with parabolic cross-section profile, illuminated by an H-polarized CSP feed was analyzed using the MAR approach. The results showed that a decrease in the inductive surface impedance due to the higher chemical potential of the graphene causes an enhancement in the antenna forward directivity. This is also consistent with the radiation pattern of the graphene reflector.

\section{REFERENCES}

[1] T. Low and P. Avouris, "Graphene plasmonics for terahertz to midinfrared applications," ACS Nano, vol. 8, no 2, pp. 1086-1101, 2014.

[2] D. Rodrigo, O. Limaj, D. Janner, D. Etezadi, F. J. García de Abajo, V. Pruneri, and H. Altug, "Mid-infrared plasmonic biosensing with graphene," Science, vol. 349, pp. 165-168, 2015.

[3] R. Depine, Graphene Optics: Electromagnetic Solution of Canonical Problems, IOP Concise Physics, Morgan and Claypool Publ., 2016.

[4] G. W. Hanson, "Dyadic Green's functions and guided surface waves for a surface conductivity model of graphene" J. Appl. Phys., vol 103, no. 6, pp. 064302/8, 2008 .

[5] T. Oguzer and A. Altintas, "Focusing ability of a microsize graphenebased cylindrical reflector in the $\mathrm{THz}$ range illuminated by $\mathrm{H}$-polarized electromagnetic plane wave," Proc. Int. Conf. Mathemat. Methods Electromagn. Theory (MMET-2016), Lviv, 2016, pp. 232-235.

[6] T. Oguzer, A. Altintas, and A. I. Nosich, "Focusing of THz waves with a microsize cylindrical reflector made of graphene in the free space," $J$. European Opt. Soc., vol. 13, art. no 16, 2017.

[7] R. Orta, P. Savi, and R. Tascone, "The effect of finite conductivity on frequency selective surface behavior," Electromagnetics, vol. 10, no. 2, pp. 213-227, 1990.

[8] A. I. Nosich, Y. Okuno, and T. Shiraishi, "Scattering and absorption of E and H-polarized plane waves by a circularly curved resistive strip," Radio Science, vol. 31, no 6, pp. 1733-1742, 1996.

[9] D. Colton and R. Kress, Integral Equation Method in Scattering Theory, Wiley Publ., 1983.

[10] A. I. Nosich, "Method of Analytical Regularization in wave-scattering and eigenvalue problems: foundations and review of solutions," IEEE Antennas Propagat. Mag., vol. 42, no 3, pp. 34-49, 1999.

[11] A. I. Nosich, "Method of analytical regularization in computational photonics," Radio Science, vol. 51, no 8, pp. 1421-1430, 2016.

[12] T. Oguzer, A. I. Nosich, and A. Altintas, "Analysis of arbitrary conic section profile cylindrical reflector antenna, H-polarization case," IEEE Trans. Antennas Propagat., vol. 52, no 11, pp. 3156-3162, 2004.

[13] T. Oğuzer, A. Altintas, and A. I. Nosich, "Analysis of the elliptic-profile cylindrical reflector with a non-uniform resistivity using the complex source and dual-series approach: H-polarization case," Opt. Quant. Electron., vol. 45, no 8, pp. 797-812, 2013.

[14] M. V. Balaban, O. V. Shapoval, and A. I. Nosich, "THz wave scattering by a graphene strip and a disk in the free space: integral equation analysis and surface plasmon resonances," J. Optics, vol. 15, no 11, pp. 114007/9, 2013

[15] O. V. Shapoval, J. S. Gomez-Diaz, J. Perruisseau-Carrier, J. R. Mosig, and A. I. Nosich, "Integral equation analysis of plane wave scattering by coplanar graphene-strip gratings in the THz range," IEEE Trans. Terahertz Science Technol., vol. 3, no 5, pp. 666-673, 2013.

[16] T. L. Zinenko, "Scattering and absorption of terahertz waves by a freestanding infinite grating of graphene strips: analytical regularization analysis," J. Optics, vol. 17, no 5, pp. 055604/8, 2015.

[17] O. V. Shapoval and A. I. Nosich, "Bulk refractive-index sensitivities of the THz-range plasmon resonances on a micro-size graphene strip," $J$. Phys. D: Appl. Phys., vol. 49, no 5, pp. 055105/8, 2016.

[18] V. Gerasik, M. S. Wartak, A. V. Zhukov, and M. B. Belonenko, "Free electromagnetic radiation from the graphene monolayer with spatially modulated conductivity in THz range," Mod. Phys. Lett. B, vol. 30, pp.. 1650185/9, 2016.

[19] W. Fuscaldo, P. Burghignoli, P. Baccarelli, and A. Galli, "Efficient 2-D leaky-wave antenna configurations based on graphene metasurfaces," Int. J. Microw. Wireless Techn., 2017. doi:10.1017/S1759078717000459

[20] M. Born and E. Wolf, Principles of Optics, Pergamon Press, 1980.

[21] N. L. Tsitsas, C. A. Valagiannopoulos, and A. I. Nosich, "Scattering and absorption of a complex source point beam by a grounded lossy dielectric slab with a superstrate," J. Optics, vol. 16, no 10, pp. $105712 / 10,2014$ 